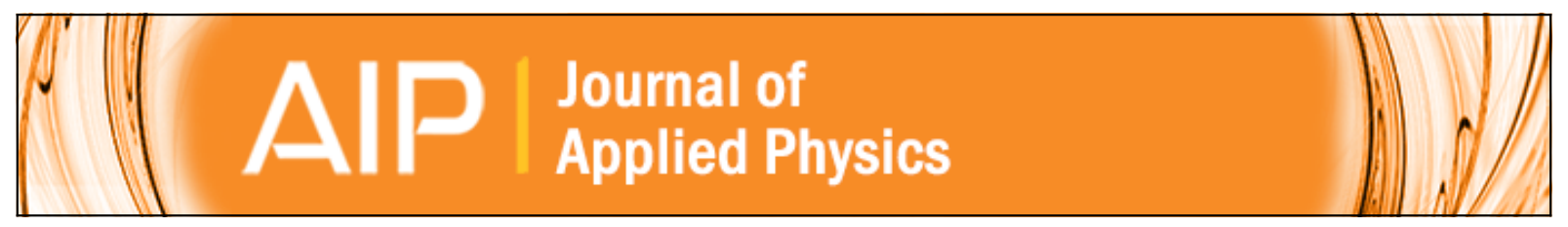

\title{
Strong enhancement of optical absorbance from ionic self-assembled multilayer thin films of nanocluster Pt and polymer dye
}

Yanjing Liu and Richard O. Claus

Citation: Journal of Applied Physics 85, 419 (1999); doi: 10.1063/1.369401

View online: http://dx.doi.org/10.1063/1.369401

View Table of Contents: http://scitation.aip.org/content/aip/journal/jap/85/1?ver=pdfcov

Published by the AIP Publishing

\section{Articles you may be interested in}

Selective growth and ordering of self-assembly on metal/polymer thin-film heterostructures via photothermal modulation

Appl. Phys. Lett. 96, 123116 (2010); 10.1063/1.3374322

Directed self-assembly of monodispersed platinum nanoclusters on graphene Moiré template

Appl. Phys. Lett. 95, 093106 (2009); 10.1063/1.3223781

Poly( $\mathrm{N}$-vinyl-2-pyrrolidone)-capped platinum nanoclusters on indium-tin oxide glass as counterelectrode for dyesensitized solar cells

Appl. Phys. Lett. 88, 103122 (2006); 10.1063/1.2186069

Self-assembled monolayer-enhanced hydrogen sensing with ultrathin palladium films

Appl. Phys. Lett. 86, 203104 (2005); 10.1063/1.1929075

Enhanced cluster mobilities on $\mathrm{Pt}(111)$ during film growth from the vapor phase

Appl. Phys. Lett. 72, 127 (1998); 10.1063/1.120664

MIT LINCOLN

LABORATORY

CAREERS

Discover the satisfaction of innovation and service

to the nation
- Space Control

- Air \& Missile Defense

- Communications Systems \& Cyber Security

- Intelligence, Surveillance and

Reconnaissance Systems

\section{LINCOLN LABORATORY}

MassachusetTS Institute OF TeCHNOLOGY

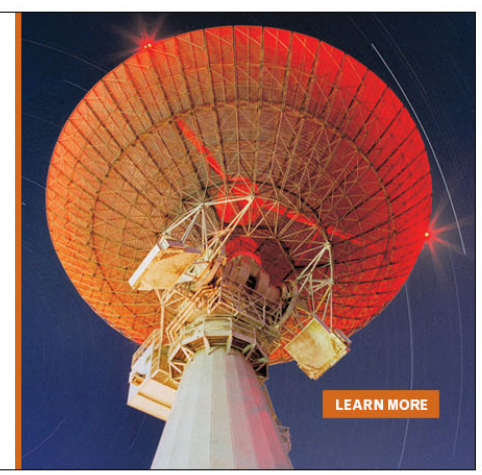




\title{
Strong enhancement of optical absorbance from ionic self-assembled multilayer thin films of nanocluster Pt and polymer dye
}

\author{
Yanjing Liu and Richard O. Claus ${ }^{\text {a) }}$ \\ The Bradley Department of Electrical and Computer Engineering, Fiber \& Electro-Optics Research Center, \\ Virginia Polytechnic Institute and State University, Blacksburg, Virginia 24061-0356
}

(Received 6 April 1998; accepted for publication 23 September 1998)

\begin{abstract}
The observation of unusually enhanced optical absorbance spectra of ionic self assembled multilayer (ISAM) thin films composed of alternating layers of Pt nanoclusters ( $<1 \mathrm{~nm}$ dimension) and polymer dye is reported. The first bilayer absorbance is found to be considerably larger than that of several succeeding bilayers even though there is no difference in composition for each bilayer. A layer-by-layer-dependent redshift in maximum wavelength position due to the strong coupling of metals and polymer molecules is observed. The saturation absorption is obtained for the first time when the required thickness of the ISAM film is deposited. The large and unusual enhancement effects are attributed to both the charge-transfer mechanism and very large local fields and collective phenomena near the surface of the small metal clusters/electrolytes and at the interfaces between the cluster/polymer multilayers. (C) 1999 American Institute of Physics. [S0021-8979(99)00601-5]
\end{abstract}

\section{INTRODUCTION}

The enhancement of surface phenomena due to microscopic surface roughness has caused a great deal of excitement during the past 2 decades. Examples are surfaceenhanced Raman scattering, ${ }^{1}$ surface-enhanced secondharmonic generation $(\mathrm{SHG}),{ }^{2}$ and enhanced photoemission from metal particles induced by inelastic electron tunneling and scanning tunneling microscopy (STM), ${ }^{3}$ to name a few. Despite substantial progress in the past, great controversy still surrounds the nature and the mechanisms of such surface-enhancement phenomena (SEP) because there is yet very little known about the detailed nature of the metal surface profile, and the adsorbed molecule bonding sites. Moreover, in nearly all prior experiments for which SEP has been observed, it is not possible to form thin films in a way that ideally emulates the desired synthesis procedure of starting off with a bare surface, and continuously varying the molecular and metal cluster coverage.

Additionally, thin films with a few monolayers of molecules adsorbed onto opaque metallic surfaces often produce strong light scattering so may not be used as active device elements in many device applications. If further applications are anticipated, in particular for optical and optoelectronic devices, it is desirable to develop new methods of SEP which do not require thick and opaque metallic films as the SEP substrate media. Moreover, the thickness of the films should vary from a few nanometers to microns to enable high resolution for various device applications.

In this article, we describe a method for the preparation of multilayer thin films alternately composed of Pt nanoclusters and polymer dye molecules by a novel ionic selfassembled monolayer (ISAM) process. ${ }^{4}$ It is demonstrated that this new process can be successfully performed with ultrafine Pt cluster $(<1 \mathrm{~nm})$ and ultrathin layers $(<1 \mathrm{~nm})$ of

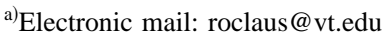

polymer dye molecules. The ultraviolet/visible (UV/vis) absorption intensity of the ISAM Pt/polymer films was found to rapidly increase as more layers were deposited, and some unique characteristics of SEP are first observed due to both the unique multilayer architecture and molecular structures, as well as nanoscopic roughness effects. Our team has demonstrated the synthesis of more than 1200 ISAM layers with excellent surface morphology, although the enhancement mechanisms for such film systems need additional analysis, the resulting thin films may be used as active device elements due to their strong SEP effects and versatile architecture, because they do not scatter much light, and because their thickness may be varied from a few nanometers to micrometers with angstrom resolution.

\section{EXPERIMENT}

Polyelectrolyte poly (diallyldimethylammonium chloride) (PDDA) was obtained from Aldrich, and polymer dye, poly R-478 (R478) from Sigma as shown in Fig. 1. Platinum nanoclusters protected by PDDA surface passivation coatings were prepared by the hydrogen reduction method. Potassium tetrachloro platinate $\left(\mathrm{K}_{2} \mathrm{PtCl}_{4}\right.$, Aldrich) dissolved in aqueous solution containing PDDA with a molar ratio of $\mathrm{Pt}$ polymer of 1:5 was reduced by bubbling first with argon, and then hydrogen gas, vigorously, each for $15 \mathrm{~min}$. A change of color from light yellow to dark brown was immediately observed. The PDDA-coated Pt cluster size in the dispersion is less than $1 \mathrm{~nm}$ as by particle size measurement (Zetasizer 3000, Malvern Instruments), and confirmed by ellipsometry measurement (AutoEL II-3W, Rudolph Technologies Inc.). Extensive ellipsometry measurements and atomic force microscopy (AFM) visualization of the growth of similar multilayer films formed by the ISAM process suggest partial interpenetration between successive molecular layers. ${ }^{4}$ Thus, the effective contribution of each bilayer to the total thickness of the thin film is about $2 \mathrm{~nm}$, as indicated by the 
<smiles>CCC(=O)C1C(=O)Nc2c(C)cc(NC(C)C(C)(C)C)c3c2C1c1ccccc1C(=O)C3C(C)(C)C(C)(C)C(C)NC(C)=O</smiles>

(a) Poly R-478

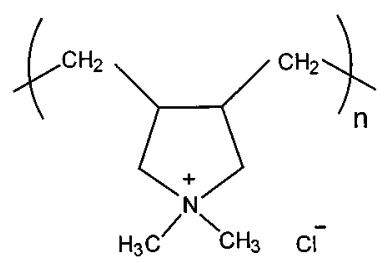

(b) Poly(diallylmethylammonium Chloride) (PDDA)

FIG. 1. Chemical structures of poly R-478 and poly(diallylmethylammonium chloride).

data in Figs. 4 and 5. This is consistent with the optical scattering measurement of Pt particle size less than $1 \mathrm{~nm}$. The concentrations of the anionic and cationic solutions are the same and $4 \times 10^{-3} \mathrm{M}$.

The typical fabrication of PDDA/R478 and Pt:PDDA/ R478 multilayer thin films using the electrostatic selfassembly process was carried out as follows. ${ }^{4}$ A precleaned quartz substrate was immersed in the cationic solution of Pt:PDDA for 3-5 min, then rinsed extensively with water. Subsequently, the slide was dipped into the anionic solution of polymer dye, R478 for 2-3 min followed by extensive washing with water. By repetition of this simple two-step process in a cyclic fashion, the layer-by-layer ionic selfassembly of consecutive layers of Pt nanoclusters and polymer dye molecules into multilayer thin films on both sides of the substrate was achieved.

UV-Vis spectroscopy (Hitachi U-2010) was used to monitor the layer-by-layer deposition process. The regular increase in the absorption spectra is due to the layer-by-layer deposition process. Consecutively alternating multilayer assemblies were also characterized by using an AutoEL II-3W ellipsometer (Rudolph Technologies, Inc.). Thickness measurement accuracy was $\pm 0.1 \mathrm{~nm}$ and each reported value was the average of at least ten measurements taken at different locations.

\section{RESULTS AND DISCUSSION}

The achievement of an ordered arrangement of multilayer films of metal nanoclusters and other molecules is very important in light of future applications and full understanding of the surface-enhanced phenomena. The fabrication of thin films of nanocluster metals and various functional molecules, with excellent molecular-level uniformity and precise control of film thickness at the angstrom levelthrough processing at ambient conditions-is highly desirable, especially for nanoclusters with small size, narrow size

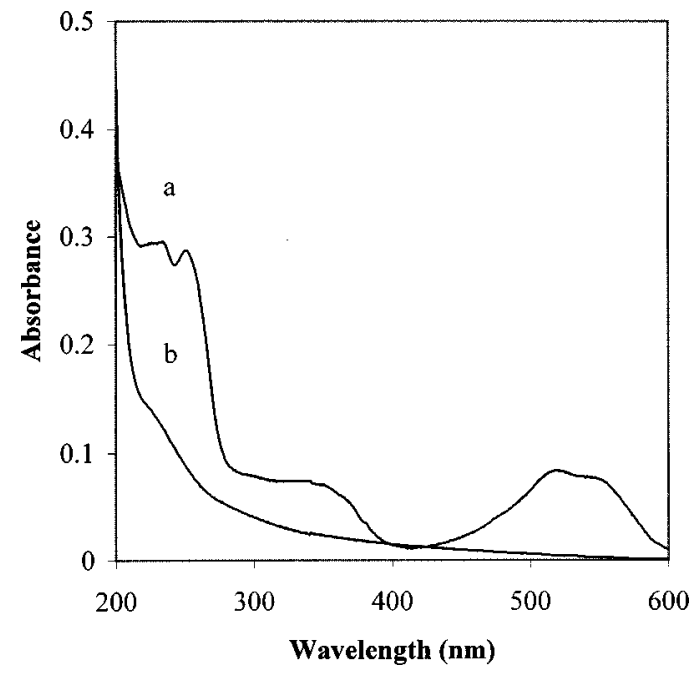

FIG. 2. UV/vis absorption spectra of poly R-478 (a) and poly(diallylmethylammonium chloride) (b) aqueous solutions.

distributions, and long-term stability. Wang and Kerker ${ }^{5}$ reported that the enhanced absorption of dye-coated particles depends on the particle size, the optical properties of the core (silver, gold, and dielectric), the coating thickness, the spheroid eccentricity, and the interaction of metal particles with adsorbed dye molecules. Glass et al. ${ }^{6}$ also found the strong coupling between the metal and the dye that has absorption bands in the same frequency range as the plasma frequency of the particle, and that this strong coupling can greatly modify the plasma resonance of the particles. In order to exhibit strong enhancement of absorbance, judicious selection of the molecules and synthesis of the extremely small particles for different applications should be considered.

The UV-Vis absorbance spectra of the R478 and (Pt:PDDA) aqueous solutions are illustrated in Fig. 2. The absorption maximum of the R478 occurs at 234, 251, 350, 519, and $550 \mathrm{~nm}$, respectively. A shoulder at $236 \mathrm{~nm}$ appears in the case of the absorption spectrum of Pt:PDDA.

Optical absorption spectra that progressively follow the deposition process and growth of PDDA/R478 ISAM films, up to 80 bilayers on quartz slides, are shown in Fig. 3. One can clearly see that the consecutive adsorption of layers is stepwise and the deposition process is very consistent from layer to layer. The linear increase in absorbance at $524 \mathrm{~nm}$ with the number of bilayers suggests that each layer adsorbed contributes an equal amount of material to the thin film, ${ }^{4}$ and yields an average optical density of 0.0058 \pm 0.0007 per bilayer at $524 \mathrm{~nm}$. Optical ellipsometry was used to monitor the growth in the thickness of the PDDA/ R478 multilayer ISAM films. A linear increase of the thickness with the number of bilayers of the PDDA/R478 ISAM films within a $\pm 3 \%$ error is demonstrated in Fig. 4 . The odd number corresponds to the PDDA layers, whereas the even one corresponds to the R478 layers. In each layer, approximately $7.2 \AA$ of R478 and 5.8 $\AA$ of PDDA were adsorbed.

The progress in the electrostatic self-assembly of organic films led us to explore the self-assembly of metal nanocluster multilayers. Here the key issue is whether metal nanoclusters can be organized into macroscopic surfaces that have well- 


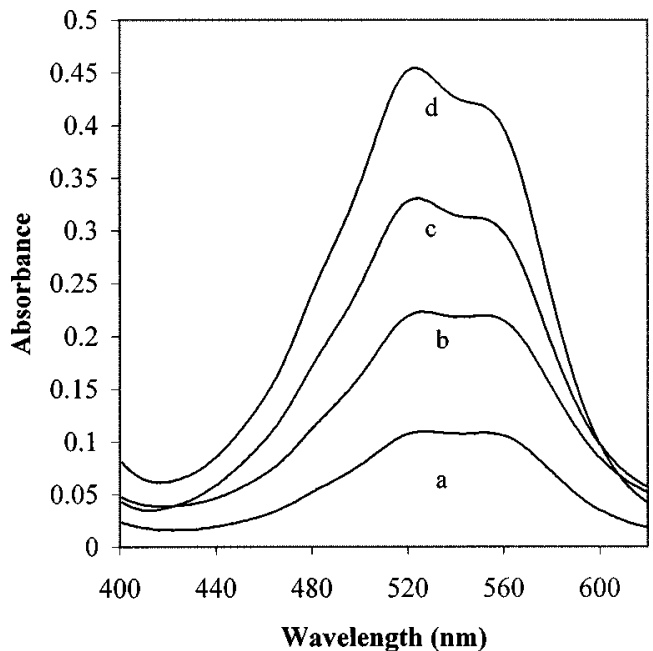

FIG. 3. Optical absorption spectra of PDDA:poly R-478 films with different bilayers on glass substrates: (a) 20 bilayers, (b) 40 bilayers, (c) 60 bilayers, and (d) 80 bilayers.

defined and uniform nanometer-scale architecture. ${ }^{7}$ Direct measurements of the increase in the thickness of the grown ISAM films of Pt:PDDA/R478 by ellipsometry is shown in Fig. 5. A linear increase of the thickness with the number of bilayers of the Pt:PDDA/R478 ISAM film within a $\pm 5 \%$ error is observed. In comparison to the $7.2 \AA$ approximate thickness of R478 in Fig. 4, the thickness of the PDDAcoated Pt cluster layers is very small, and on the order of $12.9 \AA$. The thickness of the resulting thin film as a function of bilayers thus increases in $20 \AA$ increments per bilayer, indicating very uniform Pt cluster diameters.

The absorbance spectra of the layer-by-layer increments of ISAM films of Pt:PDDA/R478 are shown in Fig. 6. The absorption spectra show two major absorption bands: one around $236 \mathrm{~nm}$ (hereafter called band A) and one around 524 $\mathrm{nm}$ (band B). Band A has a peak position similar to that of both of the Pt:PDDA complex and the R478 molecule. Band B can be attributed to R478 alone. In order to clearly compare the changes as the thin-film thickness is gradually in-

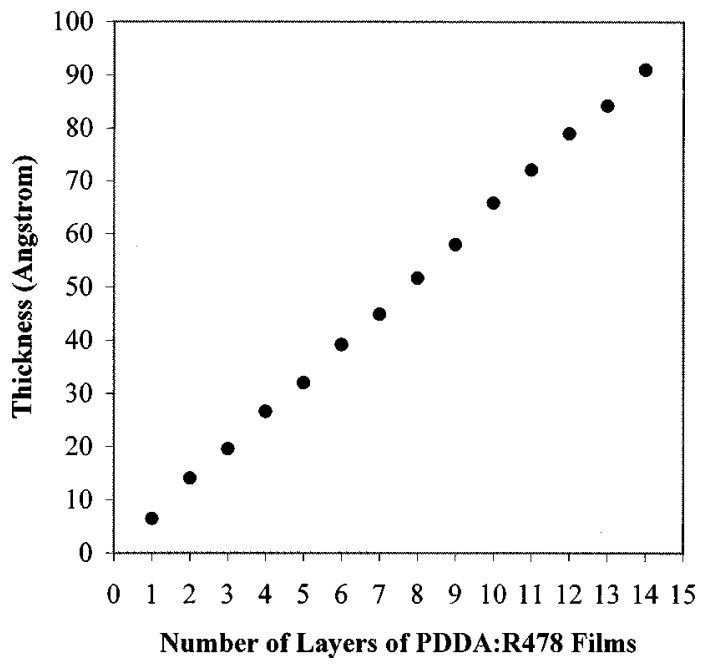

FIG. 4. Dependence of ellipsometric thickness of the adsorbed PDDA/poly R-478 films on the layer number.

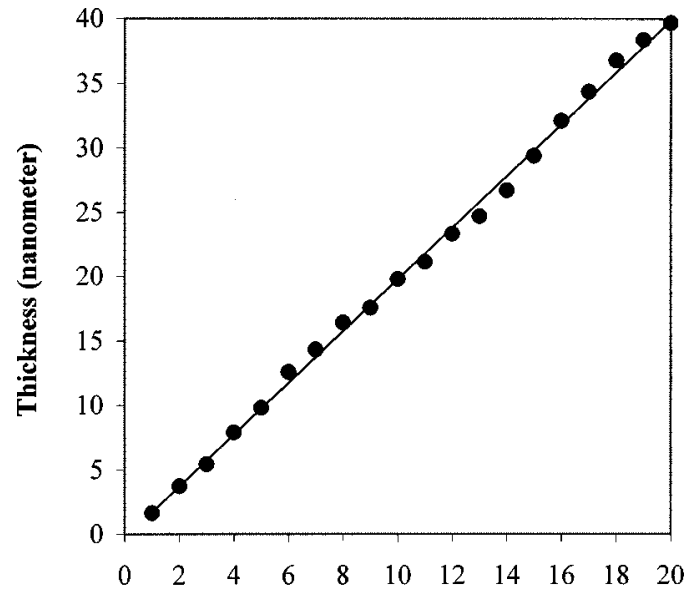

Number of Bilayers of Pt:PDDA/Poly R-478 Films

FIG. 5. Dependence of ellipsometric thickness of the adsorbed Pt:PDDA/ poly R-478 films on the layer number.

creased, the peak positions and the intensity increments in Fig. 6 are shown in Table I. For band B, the absorbance intensity of the first bilayer is 0.065 , which is about ten times larger than that $(0.0058)$ for the PDDA/R478 film. The absorbance intensity increases linearly at 0.011 (double that of PDDA/R478 ISAM films in Fig. 3) per bilayer for the first four bilayers, slightly more rapidly, at approximately 0.015 per bilayer for the fifth and sixth bilayers, then much more rapidly at 0.021-0.045 per bilayer for the additional multilayer coverage, whereas the absorption peak position of band $\mathrm{B}$ remains unchanged. For band $\mathrm{A}$, the single plasma resonance seen for Pt becomes a pronounced peak at around $236 \mathrm{~nm}$, and this can be attributed to the strong coupling between the Pt:PDDA complex and the R478 molecules. It is very interesting to see that the same first bilayer larger enhancement effect occurs again, whereas the intensity increment is no longer linear as that in band B. The intensity increases more quickly as additional layers are deposited and

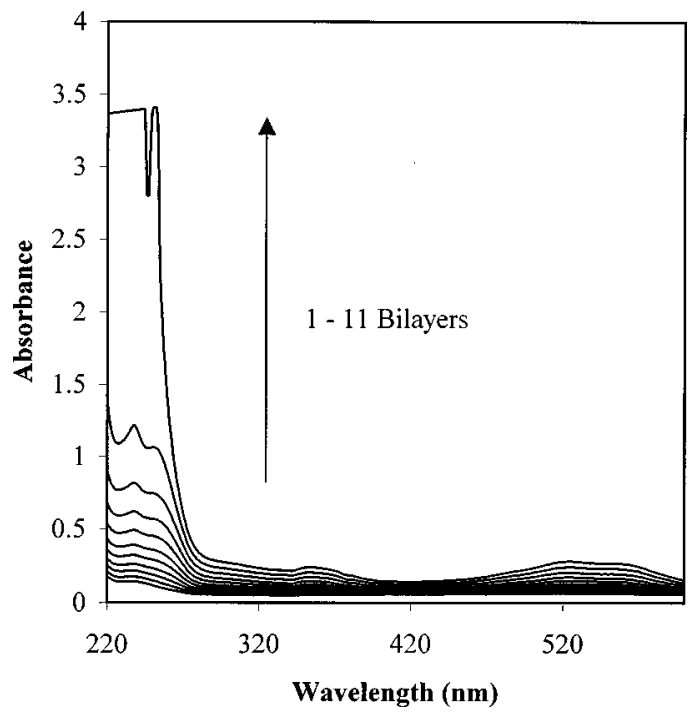

FIG. 6. UV/vis absorption spectra of Pt:PDDA/poly R-478 films up to 11 bilayers on quartz substrate. 
TABLE I. Comparisons of band positions, intensities, and intensity increments with the number of bilayers.

\begin{tabular}{|c|c|c|c|c|c|c|c|c|c|c|c|}
\hline Number of bilayers & 1 & 2 & 3 & 4 & 5 & 6 & 7 & 8 & 9 & 10 & 11 \\
\hline Band A position (nm) & 236 & 236.3 & 236.6 & 236.8 & 237 & 237.4 & 237.6 & 237.8 & 238 & 238.3 & 243 \\
\hline Intensity of band A & 0.143 & 0.176 & 0.216 & 0.261 & 0.322 & 0.392 & 0.493 & 0.622 & 0.819 & 1.215 & $3.397^{\mathrm{a}}$ \\
\hline Intensity increment $\mathrm{dA}$ & $\cdots$ & 0.033 & 0.040 & 0.045 & 0.06 & 0.07 & 0.101 & 0.271 & 0.197 & 0.396 & $2.182^{\mathrm{a}}$ \\
\hline Band B position (nm) & 524 & 524 & 524 & 524 & 524 & 524 & 524 & 524 & 524 & 524 & 524 \\
\hline Intensity of band B & 0.065 & 0.076 & 0.087 & 0.098 & 0.113 & 0.129 & 0.15 & 0.174 & 0.197 & 0.242 & 0.281 \\
\hline Intensity increment $(\mathrm{dB})$ & $\cdots$ & 0.011 & 0.011 & 0.011 & 0.015 & 0.016 & 0.021 & 0.024 & 0.023 & 0.045 & 0.039 \\
\hline
\end{tabular}

${ }^{\mathrm{a}}$ Absorption saturation is obtained.

soon reaches saturation as the 11 th bilayer is deposited. Another important feature of our enhanced absorption spectra at band A is also shown in Table I. Only for the first bilayer of the R478/Pt:PDDA film, where there is little material absorbed to the substrate to cause substantial interaction at the molecular level, does the composite film spectrum appear similar to the sum of the individual spectra of the R478 and Pt:PDDA solutions. For the thicker films (from two to ten bilayers) the maximum wavelength position continuously shifts from $236 \mathrm{~nm}$ to longer wavelengths at about $3 \AA$ per bilayer as the strong enhancement of absorbance increases very quickly with more layers deposited. It is evident that the presence of the R478 molecule affects the absorption spectrum of the Pt:PDDA nanocluster complexes greatly. Such change must be attributed to strong interaction of the metal clusters with the adsorbed molecules that have absorption bands in the same frequency range as the plasma frequency of the metal nanoclusters. ${ }^{6}$ Moreover, a large redshift of 5 $\mathrm{nm}$ is observed when the 11th bilayer is deposited, indicating that the optimal condition for absorbance enhancement is obtained. The behavior of the enhancement factor is more readily observed by taking the relative percentage increment of absorbance data in Fig. $6,\left(A_{j}-A_{i}\right) / A_{i}(i=1,2,3, \cdots, 11 ; j$ $=i+1)$ shown in Fig. 7, which gives the coverage dependence of the enhancement factor, as a function of the number of bilayers away from the first bilayer of the film. $\left(A_{j}-A_{i}\right) / A_{i}$ increases steadily at about $23 \%-26 \%$ per bilayer in the range from $n=1-8$ bilayers, quite rapidly at about $32 \%-48 \%$ per bilayer for larger values of $n=9-10$, and then approaches saturation $(180 \%)$ for $n=11$.

Direct evidence concerning the morphology and interparticle spacing and packing comes from AFM studies of ISAM Pt/polymer films. Tapping-mode AFM images were collected by using a Nanoscope III (Digital Instruments) at ambient environment. Figure 8 shows the morphology of the multilayer Pt:PDDA/R478 films with 30 bilayers on quartz substrate. Examination of the image reveals several special features: (a) the particles are closed packed, and selfassembled with each other by strong electrostatic interaction in three dimensions due to the presence of the polymer overlayers and interlayers; (b) the particle size and coverage of our film system is uniform over areas macroscopic compared to the particle size; (c) the roughness is uniform and defined solely by the Pt:PDDA diameter and the thickness of R478 monolayer as well as the repeated dipping cycles.

Although theoretical understanding of the mechanisms for SEP is not yet complete, it is generally agreed that in any given adsorbate-substrate system several enhancement mechanisms may be operating. ${ }^{8}$ However, the relative importance the various mechanisms is somewhat controversial. ${ }^{9}$

Several distinct features are observed in our absorption experiments: (a) the first bilayer absorbances at bands A and $\mathrm{B}$ were found to be considerably larger than those of a few succeeding bilayers even though there is no difference in composition for each bilayer, (b) a layer-by-layer-dependent redshift in maximum position was observed for band $\mathrm{A}$, and (c) a sharp increase in absorption enhancement only appears after several bilayer films are deposited. The very large relative percentage increments of $48 \%$ for the tenth bilayer and at least $180 \%$ for the 11 th bilayer (saturation) indicate that a resonance absorbance phenomena similar to resonance Raman scattering exists and is obtained only after a specific thickness of film is accumulated. Similar behavior is observed for multiple thin films prepared in the same way.

Potential contribution to the observed resonance due to planar optical cavity interference effects in the film have been considered. The index of refraction of the composite film material, measured by multiwavelength ellipsometry, is approximately 1.48 , so one quarter of the $236 \mathrm{~nm}$ resonance wavelength corresponds to $59 \mathrm{~nm}$ of optical path length, or $40 \mathrm{~nm}$ of distance through the film. Eleven bilayers have a thickness of approximately $22 \mathrm{~nm}$, or approximately one eighth of a wavelength. Further, as additional bilayers are added, the resonance peak at $236 \mathrm{~nm}$ is found to broaden to both shorter and longer wavelengths, and additional small absorption peaks at approximately 350 and $530 \mathrm{~nm}$ as shown

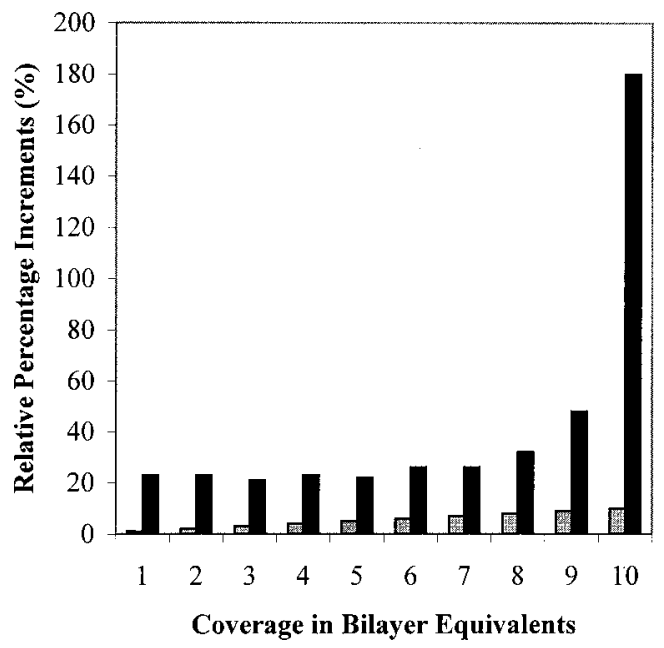

FIG. 7. Increment enhancement factor $\left(A_{j}-A_{i}\right) / A_{i}(i=1,2,3 \ldots 11 ; j=i+1)$, as a function of coverage in bilayer equivalents. 


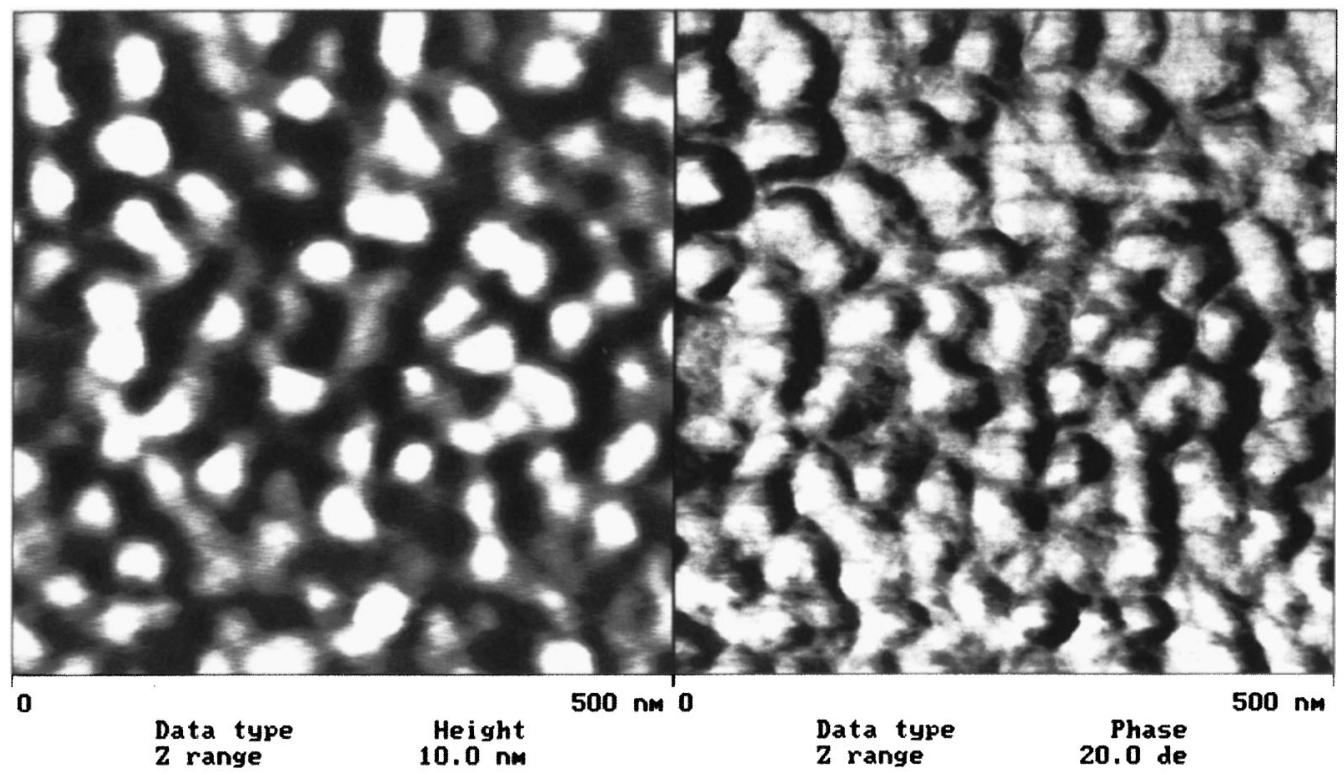

FIG. 8. Tapping-mode AFM image of 30 bilayers of (Pt:PDDA)/poly R-478 multilayer films on quartz substrate.

in Fig. 6, also appear and saturate for film optical path lengths less than a quarter wavelength. Additionally, much thicker multilayer films of two distinct materials with different indices have been intentionally designed and formed into multilayer dielectric interference filter stacks using the electrostatic energy analyzer (ESA) process. For those very different film materials and geometries, single and multilayer interference effects are clearly observed. ${ }^{10}$

On the other hand, in our film system, first, the energy structure of the extremely small Pt cluster may separate into discrete energy levels from the bulk energy band gap, and second, the metal cluster is coated with PDDA, an electrolyte. Such a metal-electrolyte interface constitutes an electric double layer that results from the generally different Fermi levels in both media. The capacitance of this electric double layer is extremely high, on the order of 50 $\mu \mathrm{F} \mathrm{cm}^{-2}{ }^{11}$ The high double layer capacitance makes the metal-electrolyte interface a place of unique experimental possibilities, which are almost impossible to be realized for a metal surface in vacuum. ${ }^{11}$ By simple charging of the double layer, high electric fields (several $10^{7} \mathrm{~V} / \mathrm{cm}$ ) and large surface charges can be easily obtained. ${ }^{10}$ For weak coupling, the charge transfer between the metal and the molecule is a small effect, and the interaction may be described as the hopping of an electron between molecule and metal. For stronger coupling the charge transfer will take place via a strong admixture of molecular orbitals and electron states in the metal, and our Pt:PDDA/R478 ISAM films meet this special requirement. When the molecules are ionically absorbed on the metal nanocluster and/or ionic molecules, the electronic excitation may be transferred to the molecule and back to the metal (and/or to ionic molecules) through the strong coupling of the metal/molecule complex. Furthermore, the layerby-layer ionic self-assembly of the selected anionic species (R478) and cationic complex (Pt:PDDA) makes the longrange charge transfer $(\mathrm{CT})$ through the multilayer structure (superlattice) possible, which may play a crucial role for the resonance absorption phenomena.

If the charge-transfer model for SEP is adopted here, when the surface of Pt:PDDA/R478 ISAM film is exposed to light, an electron of the Pt cluster is excited and transferred from its initially filled state to an empty state of a nearby molecule, i.e., first to the PDDA and then back to the Pt. At the same time, an electron from the excited anionic molecule R478 can also be attracted into the empty state of PDDA through strong ionic interaction, or may even further penetrate into the empty state vacated by the transfer of an excited electron. In this case, the layer-by-layer-dependent redshift in maximum intensity position might be simply a manifestation of the existence of a charge transfer among the ionic bonds between the layered anionic and cationic molecules and the "quasichemical bond" between the metal and PDDA complex. Thus, in solid particles of very small dimensions, photon-electron coupling will be increased, because momentum is no longer an appropriate quantum number to describe the energy transfer process. ${ }^{12}$ According to Otto et al., the charge transfer energy can thus be turned in and out of resonance with the incident photon energy. ${ }^{12-14}$ Therefore, the resonant absorption is due to the resonant coupling of the ionic molecule electronic modes and the metal's conduction electron resonance. In this metal electronmediated resonance effect, a charge-transfer transition from metal states below the Fermi level to unoccupied orbitals (called affinity levels) of adsorbates, shifted and broadened upon adsorption, is assumed. ${ }^{12}$ A possible explanation for the requirement of larger thickness for the resonant absorption saturation is that the individual Pt:PDDA/R478 complex cannot form an effective complex energy band until sufficient surface concentrations of such complex sites are reached with the buildup of ISAM films. The ISAM process thus allows the gradual filling of the total energy band structure. Counter ions, such as $\mathrm{Cl}^{-}$, may also play a role ${ }^{13}$ in absor- 
bance enhancement here. Jeanmaire and Van Duyne reported that the coadsorption of organic adsorbates and ions obviously leads to large surface-enhanced Raman scattering (SERS) intensities, however, much larger intensities occur if the anions have been replaced by halide ions. ${ }^{13}$

Although we attribute the enhancement absorption to the charge-transfer mechanism mainly based on the understanding of our experiment results and the unique multilayer-film system used, the extent to which such a charge transfer mechanism contributes to our resonant absorption is still not totally clear since the electromagnetic mechanism cannot be excluded here. There may be very large local fields and collective phenomena near the surface and at the interfacial layers of the small metal clusters/electrolytes. Such absorption enhancement may comprise both the single-particle enhancements as addressed by Gersten and Nitzan ${ }^{15}$ and collective phenomena as analyzed by Chance et al., ${ }^{16}$ Wang and Kerker. ${ }^{5,17}$

\section{CONCLUSIONS}

Layer-by-layer ionic self-assembly has been used to fabricate consecutive layers of $(<1 \mathrm{~nm}) \mathrm{Pt}$ nanoclusters and layers of polymer into multilayer. This unique multilayer structure has demonstrated a layer-by-layer-dependent redshift in maximum wavelength position, and the saturation absorption is obtained after the minimum thickness of the film is deposited. The large and unusual enhancement effects are attributed to both the charge-transfer mechanism and very large local fields and collective phenomena near the surface and at the interfacial layers of the small metal clusters/electrolytes. The above results are exciting since optical absorption plays a key role in nearly all photon-related and photon-electron-related phenomena and all spectroscopes routinely used today to characterize surfaces. In addition, the ultrathin molecular-level uniform Pt film can also be used in the catalyst industry.

\section{ACKNOWLEDGMENT}

This work was supported in part by ARO Grant No. DAAG55-97-1-0101.

${ }^{1}$ M. Fleischmann, P. J., Hendra, and A. J. McQuillan, Chem. Phys. Lett. 26, 163 (1974); D. L. Jeanmaire and R. P. J. Van Duyne, J. Electroanal.
Chem. 84, 1 (1977); M. G. Albrecht and J. A. Creighton, J. Am. Chem. Soc. 99, 5215 (1977); W. Suetaka, Surface Infrared and Raman Spectroscopy (Plenum, New York, 1995).

${ }^{2}$ C. K. Chen, T. F. Heinz, D. Ricard, and Y. R. Shen, Phys. Rev. Lett. 46, 1010 (1981); A. Wokaun, J. G. Bergman, J. P. Heritage, A. M. Glass, P. F. Liao, and D. H. Olson, Phys. Rev. B 24, 849 (1981).

${ }^{3}$ J. Lambe and S. L. McCarthy, Phys. Rev. Lett. 37, 923 (1976); R. Berndt, R. Gaisch, W. D. Schneider, J. K. Gimzewski, B. Riehl, R. R. Schlitter, and M. Tschudy, ibid. 74, 102 (1995); J. Konig, H. Schoeller, and G. Schon, ibid. 78, 4482 (1997).

${ }^{4}$ G. Decher, J. D. Hong, and J. Schmitt, Thin Solid Films 210/211, 831 (1992); M. Ferreira and M. F. Rubner, Macromolecules 28, 7101 (1995); Y. Lvov, K. Ariga, I. Ichinose, and T. Kunitake, J. Am. Chem. Soc. 117, 6117 (1995); Y. Liu, A. Wang, and R. O. Claus, J. Phys. Chem. B 101, 1385 (1997); Y. Liu, A. Wang, and R. O. Claus, Appl. Phys. Lett. 71, 2265 (1997).

${ }^{5}$ D.-S. Wang and M. Kerker, Phys. Rev. B 25, 2433 (1982).

${ }^{6}$ A. M. Glass, P. F. Liao, J. G. Bergman, and D. H. Olson, Opt. Lett. 5, 368 (1980).

${ }^{7}$ R. G. Freeman, K. C. Grabar, K. J. Allison, R. M. Bright, J. A. Davis, A. P. Gunthrie, M. B. Hommer, M. A. Jackson, P. C. Smith, D. G. Walter, and M. J. Natan, Science 267, 1629 (1995).

${ }^{8}$ R. L. Garrell, Anal. Chem. 61, 401A (1989); M. Moskovits, Rev. Mod. Phys. 57, 783 (1985); A. Otto, I. Mozek, H. Grabhorn, and W. Akemann, J. Phys.: Condens. Matter 4, 1143 (1992).

${ }^{9}$ J. Kummerlen, A. Leitner, H. Brunner, F. R. Aussenegg, and A. Wokaun, Mol. Phys. 80, 1031 (1993); T. Kume, S. Hayashi, and K. Yamamoto, Phys. Rev. B 55, 4474 (1997); F. J. Adrian, J. Chem. Phys. 77, 5302 (1982); H. Metiu, Surface Enhanced Raman Scattering (Plenum, New York, 1982), p. 1; D.-S. Wang and M. Kerker, Phys. Rev. B 24, 1777 (1981); P. N. Sanda, J. M. Warlaumont, J. E. Demuth, J. C. Tsang, K. Christmann, and J. A. Bradley, Phys. Rev. Lett. 45, 1519 (1980); J. E. Rowe, C. V. Shank, D. A. Zwemer, and C. A. Murray, ibid. 44, 1770 (1980).

${ }^{10}$ Y. Liu, R. O. Claus, Y-X. Wang, W. Zhao, and K. Lenahan, Proceedings of the Materials Research Society Conference, Boston, MA, December, 1998.

${ }^{11}$ R. Parson, Chem. Rev. 90, 813 (1990); H. Geischer, D. M. Kolb, and J. K. Sass, Adv. Phys. 27, 437 (1978); G. Valette, J. Electroanal. Chem. 122, 285 (1981); 128, 37 (1982).

${ }^{12}$ A. Otto, I. Mrozek, H. Grabhorn, and W. Akemann, J. Phys.: Condens. Matter 4, 1143 (1992).

${ }^{13}$ M. Takahashi, M. Goto, and M. Ito, Chem. Phys. Lett. 121, 458 (1984).

${ }^{14}$ B. Pettinger and H. Wetzel, Surface Enhanced Raman Scattering (Plenum, New York, 1982), p. 293; D. J. Jeanmaire and R. P. Van Duyne, J. Electroanal. Chem. 84, 1 (1977).

${ }^{15}$ J. Gersten and A. Nitzan, J. Chem. Phys. 73, 3023 (1980); 75, 1139 (1981).

${ }^{16}$ R. R. Chance, A. H. Miller, A. Prock, and R. Silbey, J. Chem. Phys. 63, 1589 (1975); Adv. Chem. Phys. 37, 1 (1978).

${ }^{17}$ D.-S. Wang and M. Kerker, Phys. Rev. B 24, 1777 (1981); D.-S. Wang and M. Kerker, ibid. 25, 2433 (1981); M. Kerker, O. Siiman, and D.-S. Wang, J. Phys. Chem. 88, 3168 (1984). 